\title{
Some physical determinants of viewing time under three instructional sets'
}

\author{
LARRY T. BROWN AMD WILLIAM FARHA \\ OKLAHOMA STATE UNIVERSITY
}

One-hundred fifty human Ss viewed 32 patterns under neutral $(\mathrm{N})$, pleasingness $(\mathrm{P})$, or interestingness (I) instructional sets. An analysis of variance indicated that patterns with larger areas were viewed longer than patterns with smaller areas under all conditions; however, this effect was more pronounced under the $\mathrm{P}$ and I conditions than under the $\mathrm{N}$ condition. Patterns containing 9-sided shapes were viewed longer than those containing 3-sided shapes under the $\mathrm{N}$ and I conditions, while the reverse was true for the $\mathrm{P}$ condition.

When given the opportunity to view a series of patterns Ss tend to look at "more irregular (MI)" patterns for longer periods of time than "less irregular (LI)" patterns (e.g., Berlyne, 1958a; Berlyne, 1958b; Berlyne \& Lawrence, 1964). However, when asked to rank-order the same patterns according to their degree of "liking" for them, Ss tend to assign higher rankings to $\mathrm{LI}$ patterns than to $\mathrm{MI}$ patterns (Berlyne \& Lawrence, 1964). In an experiment designed, in part, to examine the latter relationship further, Berlyne (1963) found that, while LI patterns received higher mean ratings of "pleasingness" than MI patterns, MI patterns received higher mean ratings of "interestingness." These findings suggest that the time spent viewing a pattern may depend, like the ranking or rating assigned the pattern, on the type of instructional set under which the $\mathrm{S}$ responds. Stated more specifically, the relation between a given pattern parameter and viewing time may depend on whether "pleasingness," "interestingness," or some other word is included in S's instructions. The present experiment was designed to study the relations between three arbitrarily selected stimulus parameters and viewing time ${ }^{2}$ under three instructional sets: a "neutral" set (NS), a "pleasingness" set (PS), and an "interestingness" set (IS)。

\section{Subjects}

The Ss were 150 undergraduate volunteers taking introductory psychology courses at Oklahoma State University. They were randomly assigned to three groups of $50 \mathrm{Ss}$ each.

\section{Stimulus Patferns}

Thirty-four $3-1 / 2$ by 4 in slides were prepared by sandwiching shapes cut from colored cellulose acetate sheets between thin plates of clear glass. Each of 32 of the slides bore a pattern representing one cell within an 8 by 2 by 2 factorial design. The three factors making up the design were Color (eight levels), Area (two levels), and Number of Turns (two levels). The two remaining slides were prepared for use as practice slides and bore patterns containing shapes varying across all levels of all three stimulus parameters.

The 32 test patterns were prepared in the following manner:

Number of Turns.

Fifty triangles and 50 9-sided polygons were constructed using Method 1 of Attneave \& Arnoult (1956). Briefly, this method consists of randomly plotting $n$ points within a matrix and connecting the points by means of a standard set of operations. The angular variability of each of the 100 shapes was next calculated according to a method proposed by Attneave (1957) ${ }^{3}$. Three triangles and three 9-sided polygons were then selected for experimental use. These six Basic Shapes were selected so that each triangle was matched in angular variability with one of the 9-sided shapes. Area.

Each triangle was equated in area with the 9-sided shape matched with it in angular variability, and each of the six shapes was then drawn with its area quadrupled, yielding a total of 12 shapes, 6 small and 6 large.

Color.

The 12 shapes were then cut from each of eight colored cellulose acetate sheets manufactured by Edmund Scientific. The eight colors were Roscolene Nos. $806,818,823,828,846,857,858$, and 874. This gave a total of 96 shapes.

The 96 shapes were arranged to form 32 patterns, with each pattern containing three shapes, either the three Basic triangles or the three Basic 9-sided polygons, of the same color and same level of area. Three shapes, rather than one, were used in each pattern so as to reduce the possible effects on viewing time of unspecified properties unique to any one given shape. Since each triangle was matched in angular variability with a corresponding 9-sided shape, all patterns were equal in their overall angular variability.

The spatial arrangement of the shapes was the same for all 32 patterns. Each of the six Basic Shapes, irrespective of color or area, was placed so that it not only occupied the same position in all patterns in which it was used but it maintained the same orientation as well. Moreover, each triangle always occupied the same position as the 9-sided shape matched with it in angular variability.

\section{Apparatus}

The patterns were projected by a lantern-slide projector equipped with a shutter controlled by a small manual switch. The S's chair was located immediately to one 
side of the projector and was separated from it by a small plywood screen which served to shield $\mathrm{E}$ and his activities from S's view.

The projection surface was a standard 4 by $4 \mathrm{ft}$ beaded screen placed approximately 62 in before S's chair. The projected area of the shapes totaled 35 in $^{2}$ for patterns containing small shapes and 140 in $^{2}$ for patterns containing large shapes.

A photoelectric cell, directed at the projection screen and connected to a standard electric timer, was located in one corner of the room, behind $\mathrm{E}_{8}$ and was not visible to $S$. The timer was placed in an adjoining room. The only illumination in the room was that provided by the projector.

\section{Procedure}

Each $\mathrm{S}$ was shown individually into the testing room, seated, and allowed about $60 \mathrm{sec}$. to adapt to the semidarkness. The $\mathrm{E}$ then read the instructions. Under neutral instructions $S$ was asked to push the switch on the word "ready," to look at the pattern for "as long as you like," and to pull back on the switch "when you don't wish to see the pattern any longer." The pleasingness and interestingness instructions were identical except that the phrase "as long as you like" was replaced with "according to how pleasing (interesting) it is to you."

The two practice slides were given first in an attempt to reduce the attentional effects of novelty, both of the general experimental situation and of the specific values of the three stimulus parameters. Inter-exposure time was approximately $10 \mathrm{sec}_{\text {; }}$, no change in inter-exposure time occurred between the last practice slide and the first test slide so that $\mathrm{S}$ received no cue signaling the beginning of the test series. The 32 test slides were presented in a different random order for each of the
50 Ss receiving the same set of instructions, but the same orders were used for all three groups.

The illumination time for each slide was recorded in tenths of seconds by a second $\mathrm{E}$ who sat before the timer in the adjacent room. An experimental session lasted approximately $20 \mathrm{~min}$.

\section{Results}

Thirty-two exposure times were recorded for each of the $150 \mathrm{Ss}$, making a total of 4800 observations. The exposure times were analyzed by means of an analysis of variance, with the data arranged in a 3 by 8 by 2 by 2 factorial design with repeated observations on the last three factors, the three stimulus factors. To avoid making untested assumptions regarding the covariances (see, e.g., Winer, 1962, pp. 369-371), the critical values for the Conservative test (Greenhouse \& Geisser, 1959, cited by Winer, 1962, pp. 305-306, 322) were used for the within-subject tests.

The mean viewing times under the three instructional sets were $6.8 \mathrm{sec}$. for the neutral set, $4.4 \mathrm{sec}$. for the pleasingness set, and $4.4 \mathrm{sec}$. for the interestingness set. The mean viewing time for the neutral set was significantly longer than those for the pleasingness and interestingness sets, $F(2,147)=7.29, p<.01$.

Patterns containing large shapes were viewed significantly longer than patterns containing small shapes, $F(1,147)=42.89, p<.001$. However, overall viewing time was not significantly affected by either the color or the numbers of turns characterizing the shapes, $F(1,147)=2.60^{4}, p>.10$, and $F(1,147)=1.78, p>.10$, respectively. No significant interactions were found among the stimulus variables.

From the point of view of the purpose underlying the design of the present experiment, the interactions between instructional set and stimulus variables are

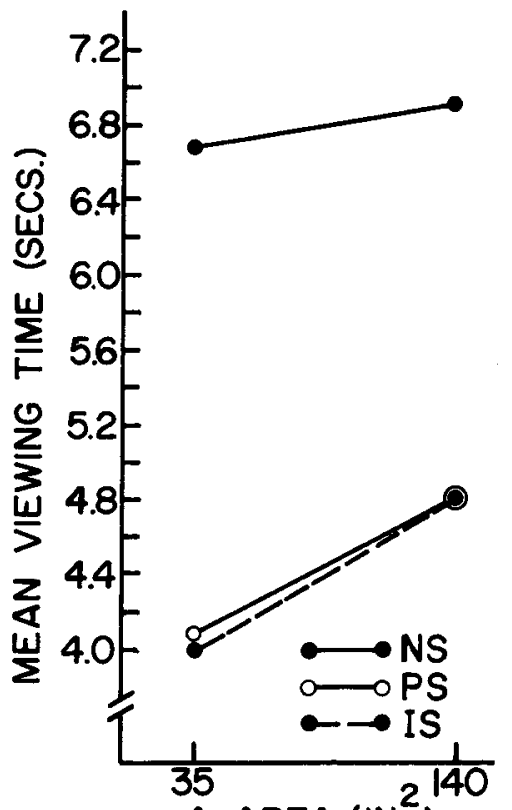

A. AREA (IN.)

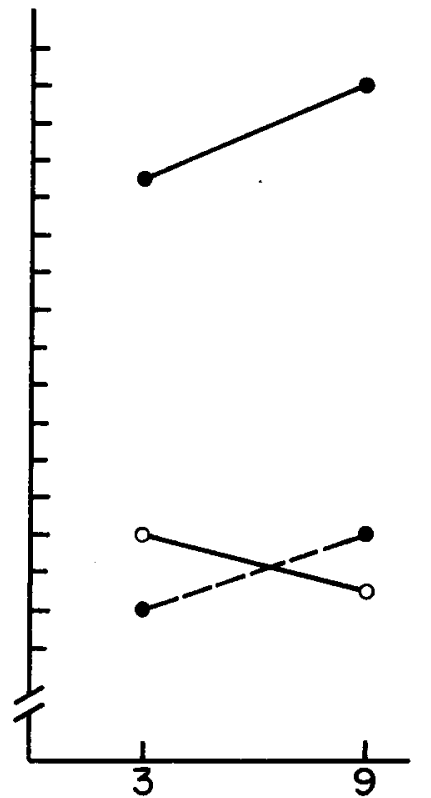

B. NO. OF TURNS
Fig. 1. Mean viewing times under neutral instructions (NS), pleasingness instructions (PS), and interestingness instructions (IS) as a function of area (Fig. 1a) and number of tums (Fig. 1b). Each point is based on the mean of 800 observations (50 Ss $x 16$ patterns). 
of particular interest. Although no significant interaction was found between instructional set and color, significant interactions we re found between instructional set and both area, $F(2,147)=4.36, p<.05$, and number of turns, $F(2,14 \hat{i})=4.03, p<.05$. The data relevant to these interactions a "e presented in Fig. 1 .

Although the large, patterns tended to be viewed longer than the smaller across all instructional conditions, it can be seen that area played a relatively greater role under the two special sets than under the neutral set (Fig. 1a). Quite a different picture emerges, however, when Fig. 1b is examined: whereas patterns containing 9-sided shapes were viewed longer than those containing 3-sided shapes under both the neutral and interestingness sets, under the pleasingness set patterns with 3-sided shapes were viewed longer than those with 9-sided shapes.

None of the higher-order interactions was found to be significant.

\section{Discussion}

As suggested by the results of Berlyne (1963) the data of this experiment indicate that the relation between a parameter of visual patterns and viewing time may depend on the instructions under which viewing occurs. The significant Instructional set by Number of Turns interaction indicates that the number of changes in contour direction may be one stimulus property whose relation to viewing time is so dependent.

With regard to the finding that, irrespective of instructions, patterns containing larger shapes were viewed longer than patterns containing smaller shapes, examination of the data reported by Berlyne \& Lawrence (1964) reveals one rather interesting exception to their overall finding that LI patterns received higher mean preference rankings than MI patterns: the MI patterns representing Variable B, "Amount of Material," received significantly higher preference rankings than the II patterns. Furthermore, while Berlyne (1963) found that the LI patterns representing most of his variables received significantly higher pleasingness ratings than the MI patterns, no significant difference was found between the LI and MI patterns of Variable B. One major dimension along which the patterns representing this variable appeared to differ was the total area occupied by the stimulus material. This, when viewed in light of the present findings regarding area, suggests that, independent of the particular words, used in the instructions, patterns of greater total area elicit both higher ratings and longer viewing times than patterns of smaller area.
While the data of this and the above-mentioned studies are insufficient to allow any conclusions regarding the nature of the motivation underlying viewing behavior, the finding that behavior under neutral instructions tends to be more positively related to behavior under interestingness instructions than to that under pleasingness instructions raises several interesting questions. For example, is it possible that simple patterns which elicit prolonged viewing when examined for their pleasingness may, in a more neutral situation, receive only the most transient inspection? Or, stated another way, do visual displays elicit prolonged viewing in neutral situations because they are interesting, and not because they are particularly pleasing (cf. Berlyne, 1963)?

\section{References}

Attneave, F. Physical determinants of the judged complexity of shapes. J. exp. Psychol., 1957, 53, 221-227.

Attneave, F., \& Amoult, M. D. The quantitative study of shape and pattern perception. Psychol. Bull., 1956, 53, 452-471.

Berlyne, D. E. The influence of complexity and novelty in visual figures on orienting responses. $J$. exp. Psychol., 1958, 55, 289296. (a)

Berlyne, D. E. Supplementary report: complexity and orienting responses with longer exposures. J. exp. Psychol., 1958, 56, 183. (b)

Berlyne, D. E. Complexity and incongruity variables as determinants of exploratory choice and evaluative ratings. Canad. $J$. Psychol., 1963, 17, 274-290.

Berlyne, D. E., \& Lawrence, G. H. Effects of complexity and incongruity variables on GSR, investigatory behavior, and verbally expressed preference. J. gen. Psychol., 1964, 71, 21-45.

Greenhouse, S. W., \& Geisser, S. On methods in the analysis of profile data. Psychometrika, 1959, 24, 95-112.

Winer, B. J. Statistical principles in experimental desion. New York: McGraw-Hill, 1962.

\section{Notes}

1. This research was supported, in part, by National Institutes of Health Grant No. PHS MH-10350-01, and administered by the Research Foundation, Oklahoma State University.

2. Duration of viewing has been used as a measure of exploratory behavior (e.g., Berlyne \& Lawrence, 1964): however, since to the authors' knowledge there has been little empirical attempt to relate viewing time to other measures of exploratory behavior, it was thought that treatment of this paper in terms of exploratory behavior might be premature.

3. Angular variability describes the extent to which adjacent angles on the contour vary in size and direction. The measurement procedure proposed by Attneave (1957) involves assigning positive values to convex angles and negative values to concave angles and subtracting the size of each angle from the size of the angle immediately preceding it on the contour. The resulting differences are then added, their sign ignored, to obtain a mean difference. This mean difference is used as a measure of angular variability. 4. It should be noted that the $F$ for color is significant at the .05 level when the critical values for the Usual test $(7,1029)$ are used. 